http://jmscr.igmpublication.org/home/ ISSN (e)-2347-176x ISSN (p) 2455-0450

crossref DOI: https://dx.doi.org/10.18535/jmscr/v8i10.25

\title{
Role of Subcutaeous Suction Drain in Reducing Surgical Site Infections in Emergency Abdominal Surgeries
}

\author{
Authors \\ Dr P.Vigneshwaran ${ }^{1}$, Dr N.Junior Sundresh ${ }^{2 *}$, Dr D.Gopikrishna ${ }^{3}$, \\ Dr R.Logesh Kumar ${ }^{4}$, Dr A.P.S.Gurupraveen ${ }^{5}$ \\ ${ }^{1,4,5}$ Post Graduate, Dept of General Surgery, Rajah Muthiah Medical College and Hospital, Annamalai \\ Nagar, Chidambaram, India, 608002 \\ ${ }^{2}$ Professor, Dept of General Surgery, Rajah Muthiah Medical College and Hospital, Annamalai Nagar, \\ Chidambaram, India, 608002 \\ ${ }^{3}$ Associate Professor, Dept of General Surgery, Rajah Muthiah Medical College and Hospital, Annamalai \\ Nagar, Chidambaram, India, 608002 \\ *Corresponding Author \\ Dr N.Junior Sundresh
}

Professor, Dept of General Surgery, Rajah Muthiah Medical College and Hospital, Annamalai Nagar, Chidambaram, India, 608002

\begin{abstract}
Background: Surgical site infection (SSI) is one of the most important and common morbidity in postoperative patients. Collection of blood and/or serous fluids in subcutaneous spaces that later gets infected is an important factor for surgical site infections in emergency laparotomies. This study compares the incidence of surgical site infection in post emergency abdominal surgical wounds with subcutaneous suction drains versus those in whom drain was not placed.

Methods: A prospective interventional study done with 50 subjects in department of surgery at rural tertiary centre. Based on exclusion and inclusion criteria patient were randomly selected for cases (with post-operative suction drain) and controls. Patients were followed up for 30 days from the date of surgery and observed for development of SSI.

Results: $16 \%$ of patients in drain group and $36 \%$ of patients in non-drain group developed infection. Incidence of SSI in no drain group was double the times than the drain group statistically.

Conclusions: Subcutaneous drain plays a significant role in reducing the incidence of surgical site infection in emergency laparotomy.

Keywords: Emergency laparotomies, Subcutaneous suction drain, Surgical site infections.
\end{abstract}

\section{Introduction}

Surgical site infection (SSI) is defined as the infection presenting within 30 days after a surgical procedure if no prosthetic is placed and up to 12 months if a prosthetic is implanted in the patients. ${ }^{1}$ Surgical infections (SSI) causes significant discomfort for patients in general surgery ward with excess morbidity and mortality, and also financial burden on the health care system. Even though some reports mention SSI as second only to urinary tract infection as the commonest hospital acquired infection. Actually SSI is most 
commonly encountered as nosocomial infection in surgical patients. ${ }^{2-4}$ The causes for developing SSI is multifactorial and include the amount of microbial contamination in the operation site which is indicated by wound classification as clean wound, clean contaminated wound, contaminated wound and dirty wound. Patient age, duration of surgery, preoperative shaving of site, hypothermia and comorbidities e.g. diabetes and obesity. ${ }^{5}$

The objective of study was to compare the incidence of surgical site infections in post emergency abdominal surgical wounds with subcutaneous suction drains versus those in whom subcutaneous drains are not used.

\section{Methods}

A prospective interventional study for consecutive 30 days on 50 subjects ( 25 drain group and 25 nodrain groups).

This prospective case series was conducted at RMMCH, Annamalai University, Chidambaram, Tamilnadu, a tertiary care centre, between December 2018 and December 2019.

All of the 50 patients admitted to emergency surgery department and underwent laparotomy within 24hrs of admission and those above 18 years are included in this study. The demographic data of the patients including alcohol and smoking history were collected and categorized based on ASA Classification. The use of prophylactic antibiotics, the time duration of surgery, clinical evaluation of wound (whether clean, contaminated or dirty) were recorded on a data sheet.

The skin incision was made using a scalpel; and using electrocautery subcutaneous fat was dissected. The wound was irrigated with 2 Litres of saline solution just before skin closure. The fascia/muscle layer was closed by using vicryl and the skin was closed by using 3-0 ETHILON. Surgical procedures between the cases and control groups were of no much difference, except that a suction drain was inserted in the subcutaneous tissue space in case group. The drain exit site was separated from the incision. The removal of suction drain was on postoperative day five.

SSI cases were diagnosed within 30 days from the date of surgery by the disease control and prevention (CDC) criteria:

- Purulent discharge in drain with or without laboratory confirmation from the incision site

- Infective organisms isolated from an aseptically obtained culture from the incision site discharge

- Any of the following symptoms/signs of infection: Pain, local swelling, erythema, or heat and superficial incision was opened by surgeon deliberately, unless the incision was culture-negative; and

- Diagnosis of s-SSI by the surgeon or ICT.

\section{Results}

Total number of patients taken into the study were 50 and were selected randomly as cases and controls. Out of these 50 patients 39 were male and 11 were female. $26 \%$ patients developed surgical site infection post emergency abdominal surgery. Out of 25 patients in the 'drain' group (cases), 21 were male and 4 were females, and 4 patients $(16 \%)$ developed surgical site infection. Out of 25 patients in the 'no drain' (controls) group, 18 were males and 7 were females, and 9 patients $(36 \%)$ developed surgical site infection. The average time of removal of suction drain were on postoperative day five (range 2-12)

There were no to minimal complications associated with the insertion of subcutaneous suction drain in study group. The incidence of surgical site infection was lower in drain group than the no drain group, which was statistically significant. Age, sex, duration of surgery, blood transfusion, diabetes, smoking and alcohol history, body mass index, blood loss during surgery, stoma, pre and post-operative albumin level were not significant. 
Table 1: Distribution of patients in the study

\begin{tabular}{|l|l|}
\hline Distribution of patients & 50 \\
\hline Total no. of patients & 39 \\
\hline Total no. of male patients & 11 \\
\hline Total no. of female patients & 25 \\
\hline No. of patients without drain (controls) & 25 \\
\hline No. of patients with drain (cases) & \\
\hline
\end{tabular}

Graph 1: Distribution of patients in the study

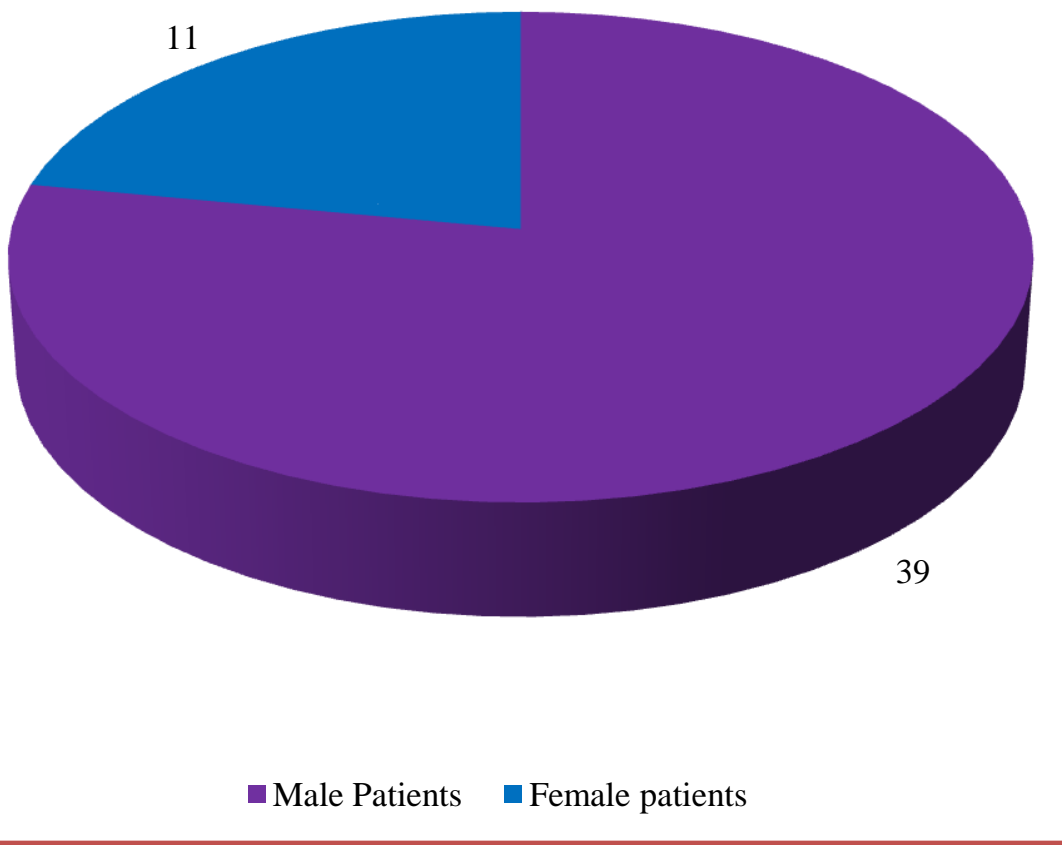

Table 2: Outcome of the study

\begin{tabular}{|l|l|}
\hline Outcomes \\
\hline \% of patients that developed infection in drain group & $16 \%$ \\
\hline$\%$ of patients that developed infection in non drain group & $36 \%$ \\
\hline Overall \% of patients that developed infection & $26 \%$ \\
\hline
\end{tabular}

\section{Discussion}

SSI is one of the most serious infectious complications of surgery. Much study proves that removing the blood and serous fluids from the wound by using drains before it get infected prevents morbidity. ${ }^{6}$ This concept is frequently implemented in clinics. However, a meta-analysis showed that prophylactic subcutaneous drainage to prevent wound complications is not efficient in gynecology. ${ }^{7}$ Abdominal surgeries mainly lower GI surgeries carry a higher risk of wound infection, over three times the average risk. ${ }^{9}$ Still there are only few reports on the efficacy of use of subcutaneous drain for the prevention of s-SSI following emergency surgery. The reason is that the incidence of s-SSI is related to amount of bacterium entering the wound, hematoma formation, potential dead space subcutaneously, disturbance of the local blood flow, and the amount of bacterias in the surgical organ. ${ }^{8}$

The use of suction drain can reduce the bacterial load in and around the wound also by removing residual blood from the wound that serves as a medium for bacterial growth. This drain is an 
active drain that uses the power of suction. After $48 \mathrm{hrs}$ the luminal obstruction of such drains increases and fluid drainage becomes poor. ${ }^{8}$

Numerous risk factors are there for developing a SSI. smokers are at a 30\% increased risk of SSI after major colorectal procedures and cessation of smoking reduces SSI. ${ }^{10,11}$ obesity have also been linked to increased risk of SSI in some procedures rising from $7 \%$ up to $23 \% .^{12,13}$ Depth of the subcutaneous fat has been a strong risk factor for SSI and has shown to be a useful predictor for SSI risk. ${ }^{14,15}$ Many other factors likepoor nutrition and poor diabetes control, certain comorbidities, ASA class, and time duration of surgery has been identified as important factors affecting SSI. ${ }^{15,16}$

Many interventions have been proposed to reduce SSIs and are used in routine practice. Hand washing, by minimising shaving of the surgical site, and preoperative antibiotics have all gained acceptance in the surgical community. ${ }^{17}$ But the usage of drains after surgery however has declined in recent times. Its been shown that drains provide no advantage after cholecystectomies, inguinal hernia surgery, and various types of surgery. ${ }^{18}$ Usage of drains after abdomino-perineal excision of rectum and repair of incisional hernias are still popular due to inconclusive evidence and surgeon preference. ${ }^{19}$ Drains are used in some of the major plastic surgery procedures as they are believed to reduce collections in closed spaces. ${ }^{20}$

Its been understood that the presence of hematoma, serous fluid, and dead space in surgical incisional wounds increases the infection risk as they can act as culture medium. ${ }^{21}$ In these cases Subcutaneous suction drains can reduce the risk of infection. However, the use of subcutaneous wound drainage postoperatively is not accepted globally as they can cause discomfort and increases the hospital stay.

The National Emergency Laparotomy Audit (NELA) a new initiative in the United Kingdom to audit and subsequently reduce complications after emergency abdominal surgeries. ${ }^{22}$ SSIs are still a major concern after emergency abdominal surgeries and within the remit of NELA. This highlights the importance of interventions needed to reduce SSI in emergency abdominal surgeries. Fujii et al, included high risk patients like those undergoing emergency abdominal surgery, and those with thicker subcutaneous fat and found out that the risk ratio reduces in the SSI rate in the drain group (RR 0.37 (0.15-0.9). ${ }^{15}$ Imada et al, showed no significant reduction in SSI incidence when using a drain in all surgical patients but there were a reduction in SSIs in the high risk patients from $15 \%$ to $8 \%$. Its been reported by Soper et al, that thicker subcutaneous fatis an independent risk factor for SSI.14 It shows that the subcutaneous suctions drains may be beneficial in high risk patients but it is not evident in the meta-analysis due to under powering.

This study shows that subcutaneous suction drain placement reduces the SSI significantly in all emergency abdominal surgeries.

\section{Conclusion}

Subcutaneous suction drains in emergency laparotomies play significant role in reducing the surgical site infection incidence by more than $50 \%$.

Funding: No funding sources

Conflict of interest: None declared

Ethical approval: The study was approved by the institutional ethical committee

\section{References}

1. Awad SS, Palacio CH, Subramanian A, Byers PA, Abraham P, Lewis D, et al. Implementation of a methicillin-resistant Staphylococcus aureus (MRSA) prevention bundle results in decreased MRSA surgical site infections. Am J Surg, 2009; 198: 607-10.

2. Kirby JP, Mazuski JE. Prevention of surgical site infection SurgClin N. 2009; 89: 365-89.

3. Edwards PS, Lipp A, Holmes A. Preoperative skin antiseptics for preventing surgical wound infections after 
clean surgery. Cochrane database. Syst Rev. 2004: CD003949.

4. Homer-Vanniasinkam S. Surgical site and vascular infections: treatment and prophylaxis. Int J Infect Dis. 2007; 11(1):17-22.

5. Mangram AJ, Horan TC, Pearson ML, Silver LC, Jarvis WR.Guidelines for prevention of surgical site infection. Infect Control HospEpidemiol. 1999; 20:250-78.

6. Baier PK, Glück NC, Baumgartner U, Adam U, Fischer A, Hopt UT. Subcutaneous Redon drains do not reduce the incidence of surgical site infections after laparotomy. A randomized controlled trial on 200 patients. Int J Colorectal Dis. 2010; 25:639-43.

7. Hellums EK, Lin MG, Ramsey PS. Prophylactic subcutaneous drainage for prevention of wound complications after cesarean delivery-a metaanalysis. Am J Obstet Gynecol. 2007; 197: 22935.

8. Numata M, Tanabe H, Numata K, Suzuki Y, Tani K, Shiraishi R, et al. The efficacy of subcutaneous penrose drains for the prevention of superficial surgical site infections. Jpn J Gastroenterol Surg. 2010; 43: 221-8.

9. Watanabe A, Kohnoe S, Shimabukuro R, Yamanaka T, Iso Y, Baba H, et al. Risk factors associated with surgical site infection in upper and lower gastrointestinal surgery. Surgery Today. 2008; 38(5):404-12.

10. Sharma A, Deeb AP, Iannuzzi JC, Rickles AS, Monson JRT, Fleming FJ. Tobacco smoking and postoperative outcomes after colorectal surgery. Ann Surg. 2013;258(2):296-300.

11. Sørensen LT. Wound healing and infection in surgery. The clinical impact of smoking and smoking cessation: a systematic review and metaanalysis. Arch Surg. 2012; 147(4):373-83.
12. Kwaan MR, Sirany AME, Rothenberger DA, Madoff D. Abdominal wall thickness: is it associated with superficial and deep incisional surgical site infection after colorectal surgery? Surg Infect. 2013; 14(4):363-8.

13. Van Walraven C, Musselman R. The surgical site infection risk score (SSIRS): a model to predict the risk of surgical site infections. PLoS ONE. 2013;8(6):Article ID e67167.

14. Soper DE, Bump RC, Hurt WG. Wound infection after abdominal hysterectomy: effect of the depth of subcutaneous tissue. Am J Obstet Gynecol. 1995;173(2):46571.

15. Fujii T, Tsutsumi S, Matsumoto A. Thickness of subcutaneous fat as a strong risk factor for wound infections in elective colorectal surgery: impact of prediction using preoperative CT. Digest Surg. 2010;27(4):331-5.

16. Cheadle WG. Risk factors for surgical site infection. Surg Infect, 2006;7:

S711.

17. Diana M, Hübner M, Eisenring $M C$, Zanetti G, Troillet N, Demartines N. Measures to prevent surgical site infections: what surgeons (should) do. World J Surg. 2011; 35(2):280-8.

18. Kosins AM, Scholz T, Cetinkaya M, Evans GRD. Evidence-based value of subcutaneous surgical wound drainage: the largest systematic review and metaanalysis. Plastic Reconst Surg. 2013;132(2):443-50.

19. Bohnen JMA. Use of drains in Abdominal Wall Hernias: Principles and Management, R. Bendavid, Ed, Springer, New York, NY, USA; 2001:328.

20. He XD, Guo ZH, Tian JH, Yang KH, Xie $\mathrm{XD}$. Whether drainage should be used after surgery for breast cancer? A systematic review of randomized 
controlled trials. Med Oncol. 2011; 28: S22-30.

21. Chelmow D, Rodriguez EJ, Sabatini MM. Suture closure of subcutaneous fat and wound disruption after cesarean delivery: a meta-analysis. Obstetrics Gynecol. 2004; 103(5):974-80.

22. Saunders DI, Murray D, Pichel AC, Varley $\mathrm{S}$, Peden CJ. Variations in mortality after emergency laparotomy: the first report of the UK emergency laparotomy network. Br J Anaesth. 2012;109(3):368-75. Imada $S$, Noura S, Ohue M. Efficacy of subcutaneous penrose drains for surgical site infections in colorectal surgery. World J Gastro Surg. 2013; 5(4):110-4. 